\title{
Language and Social Competence: An Integrated Approach to Intervention
}

The editors and editorial staff of Seminars in Speech and Language invite and welcome suggestions for issues of particular concern to practicing clinicians. For some time, there have been several requests that we offer an issue to address the social consequences of developmental language disorders. I am pleased to present this excellent treatment of the subject, ably and graciously coordinated by Guest Editors Bonnie Brinton and Martin Fujiki. In this issue, researchers and clinicians explore the social impact of language impairment across a variety of diagnostic conditions, and offer directed and practical suggestions for targeting areas of social functioning that are adversely impacted by linguistic impairment and for remediating them. Across articles, a primary concern is echoed that we do not fully assist children with impairments in language comprehension or production unless we provide them with the functional skills necessary to interact with their peers across the wide variety of educational and social settings that they will encounter in everyday life. Learning to produce grammatical utterances is not enough. Guidelines presented in this issue can guide clinicians in assisting these necessary and critical functional gains in communication.

In addition, we have received several requests over the years to address selective and elective mutism, a condition that does not receive much attention in the speech-language pathology literature. Because mutism was a logical fit to the major conceptual thrust of this issue, I wish to specifically thank Alison McInnes and Katharina Manassis, of the Hospital for Sick Children, Toronto, Canada, for answering my request for their help with an excellent treatment of this subject specifically targeted for practicing SLPs.

As usual, we believe that this issue provides a coherent and topical treatment of a topic of substantive interest to professionals in our discipline, and we welcome comments and feedback, as well as additional suggestions for future volumes that can help to update and deepen skills in your assessment and treatment of individuals with communicative impairments.

Nan Bernstein Ratner, Ed.D. Co-Editor in Chief ${ }^{1}$ 